\title{
Management of recurrent retinal detachment in silicone oil filled eyes: keys to diagnosis of pathogenesis and management
}

\begin{abstract}
The use of vitrectomy techniques, augmented with silicone oil tamponade, signifies an important shift in the management of complicated retinal detachments. Recurrence of retinal detachment under silicone oil provides both a diagnostic challenge for the pathogenesis and for how to manage. Unfortunately, no study has clearly put guidelines for the diagnosis and management of these complicated cases. In this article we review what was provided to us in the literature from the experience of other surgeons in addition to our own experience in the past 20 years in the management of these cases.
\end{abstract}

Keywords: recurrent detachment, silicone oil, pathogenesis, management
Volume I Issue I - 2014

\author{
Ahmed Abd Allah Ibrahim Darwish \\ Professor of ophthalmology Ain Shams University Cairo, Egypt \\ Correspondence: Ahmed Abd Allah Ibrahim Darwish, \\ Professor of ophthalmology, Ain Shams University, I 9 Sibawih \\ Elmasry, St. Nasr City Cairo, Egypt, Tel 0568568290 , \\ Email ahmed_darwish33@mail.com
}

Received: August 06, 2014 | Published: August 09, 2014

\section{Abbreviations: PVR, proliferative vitreoretinopathy}

Causes of redetachment of the retina in silicone-oil filled eyes

Several factors are involved in the pathogenesis of recurrence of retinal detachment under silicone oil, these include:

\section{Proliferative vitreoretinopathy (PVR)}

i. The most common reason for redetachment in silicone oil-filled eyes is PVR, and it occurs in approximately $80 \%$ of the affected eyes. ${ }^{1,2}$

ii. It is known that the tamponade force of silicone oil is less than that of air, and the oil does not have any negative effect on PVR. ${ }^{3,4}$ Instead, Silicones recompartmentalize at the retinal surface (and iris surface) increasing reproliferation at these sites because of retention of cells and cell-cell interaction factors, this causes posterior and anterior PVR respectively. The silicone oil globule in the eye, being lighter, floats; therefore, the lower half of the retina does not have a direct contact with the silicone oil meniscus. This probably confines the posterior PVR process, in most instances, to the lower retina. ${ }^{5}$

\section{Leaking retinal break}

The redetachment could be because of a leaking retinal break in the far periphery, mostly inferior, and therefore not supported by the oil globule, with no PVR. ${ }^{6}$

\section{Intrinsic retinal contraction}

In some eyes, the retina comes off because of an intrinsic retinal contraction with no obvious preretinal or subretinal proliferation. ${ }^{6}$

\section{Subjects and methods}

In our series of 50 cases of recurrent retinal detachment under silicone oil (not including cases of proliferative diabetic retinopathy or giant breaks) we had 41 cases caused by PVR either posterior focal PVR
(21 cases) or posterior diffuse $(13$ cases $)$ or a combination of anterior and posterior PVR (7 cases). 9 cases had a lower break not associated with PVR and of these 3 had additional intrinsic contraction. It was noticed that all cases with recurrent detachment without PVR or those with posterior PVR were confined to the lower half of the retina. Cases with a combined anterior and posterior PVR were more aggressive and involved a total or near total retinal detachment (Figure 1).

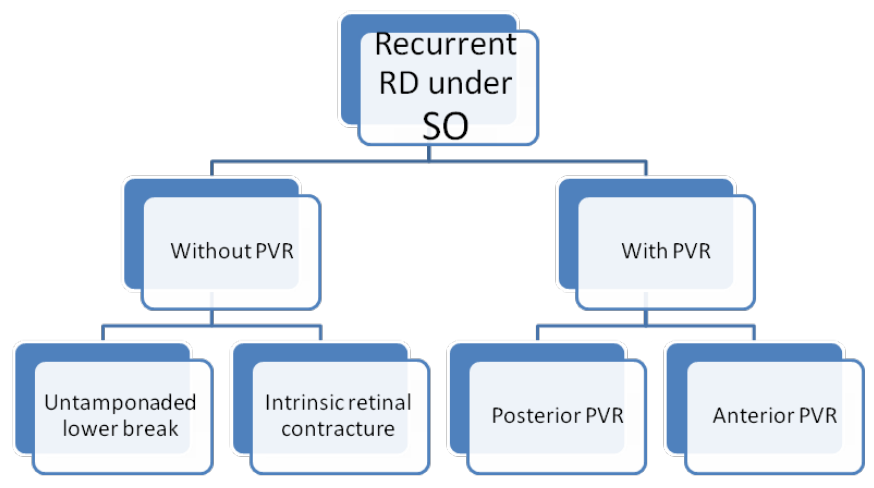

Figure I Causes of recurrent retinal detachment under silicone oil.

A summary of these factors is in Figure I

\section{Management}

Management of these cases depended, in our study, on whether a PVR was present or not (Figure 2).

\section{Techniques used in our study}

\section{Silicone oil not supporting a lower break without PVR}

Face down position and laser: In cases where there was an unsupported lower break without PVR the patient was asked to sleep in a face down position (or, alternatively, sit leaning forward on a table in a face down position)as long as possible, and the flattening of the 
lower retina was observed. If the retina flattened completely, several raws of laser were used to surround the break and the patient was asked to continue the face down position until the laser marks began to darken. This technique was successful in 3 of the 6 cases where there was no intrinsic contraction of the retina.

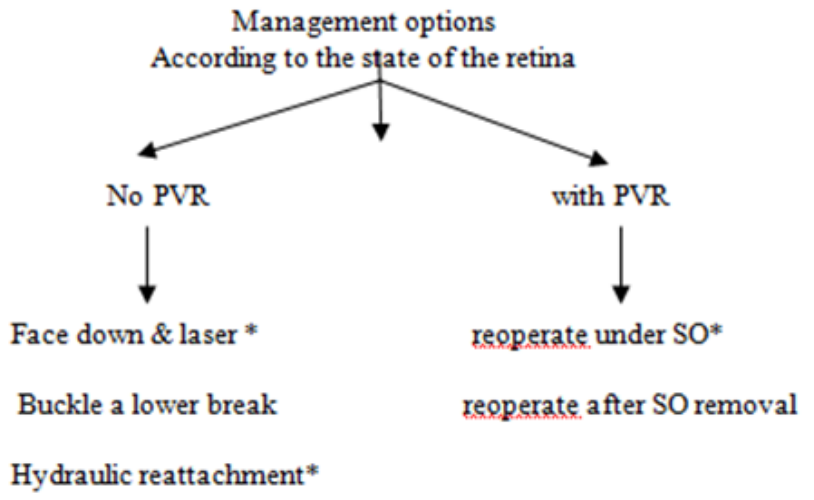

Figure 2 Management options for cases of recurrent retinal detachment under $\mathrm{SO}$

Scleral buckle: Only reserved for small lower breaks with no PVR to improve their contact and tamponade with the silicone oil bubble. Cryo is added around the break. We did not use this technique in any of our cases although it was mentioned to be effective by other authors.?

Hydraulic reattachment of the retina: (Figure 3) Silicone oil fluid exchange must be done slowly with:

i. Flute needle put in the retinal break in the fluid meniscus.

ii. Observe the fluid-silicone oil interface over the flute needle shaft as it goes down towards the retina.

iii. Observe the arterial pulsations over disc to avoid excessive increase in IOP \& SO passing under the retina if residual traction was present.

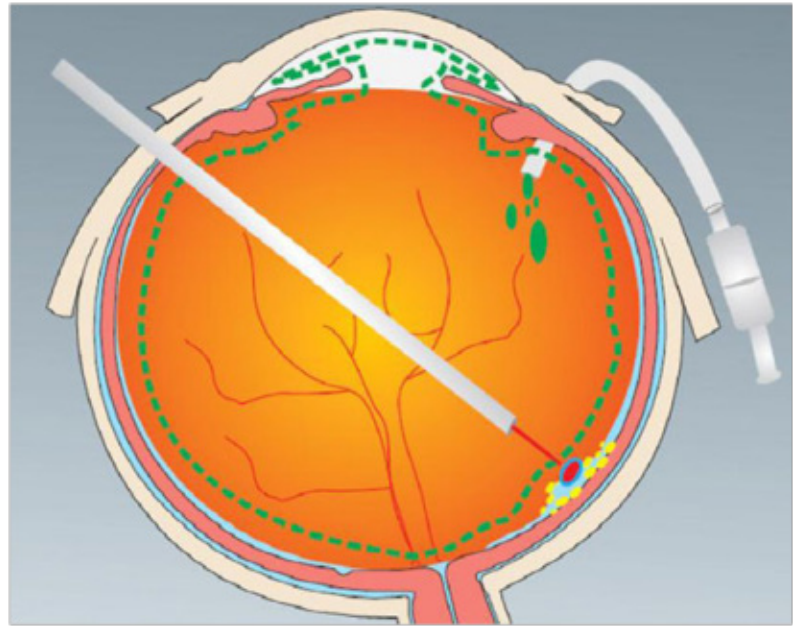

Figure 3 Hydraulic reattachment of the retina.

This technique was used as a theraputic measure in case of failure of face down position, either due to patient non compliance ( 2 cases) or viscid sub-retinal fluid ( 1 case). The technique was also useful to diagnose intrinsic contraction of the retina where the retina remained elevated and in some cases the retinal break enlarged gradually as the silicone oil was injected. In these cases, care must be taken to stop silicone oil injection otherwise it will go under the retina. A retinotomy was done in those 3 cases.

\section{Lower retinal break with intrinsic retinal contraction or PVR}

Reoperation under silicone oil: This technique was used in cases of posterior PVR not associated with anterior PVR (34 cases) and also for cases associated with anterior PVR ( 7 cases).

\section{The following are the steps we used during surgery}

Insertion of infusion cannula (Figure 4)

i. Infusion cannula is connected to a syringe filled with silicone oil

ii. Be sure that no air is present in the tubings

iii. Fix the infusion cannula in place $\&$ check the freedom of its tip in the vitreous cavity (Figure 5).

Clearing obstacles hindering posterior segment visualization:

i. Emulsified oil in AC, (Figure 5).

ii. Displace it to the sides of AC using healon.

iii. Do not attempt to do suction using a syringe and avoid AC hypotony to avoid the passage of silicone oil from the posterior segment to the anterior chamber.

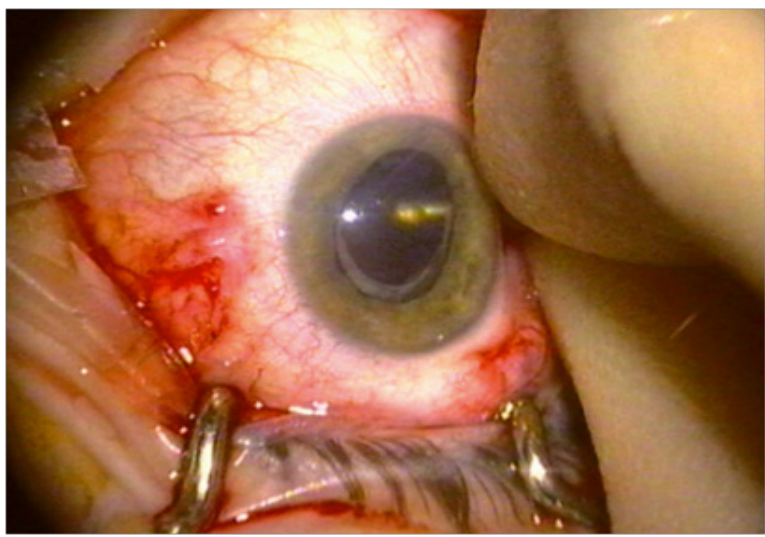

Figure 4 Insertion of infusion cannula and checking that its tip is free in the vitreous cavity

\section{Cataract (Figure 6)}

i. Phacoemulsification \& foldable IOL in the bag

ii. Keep the AC pressure always higher than the posterior segment.

iii. Avoid extensive dialing of the IOL.

iv. Keep the anterior chamber filled with healon to avoid passage of silicone oil to the anterior chamber during posterior segment work (Figure 7). 


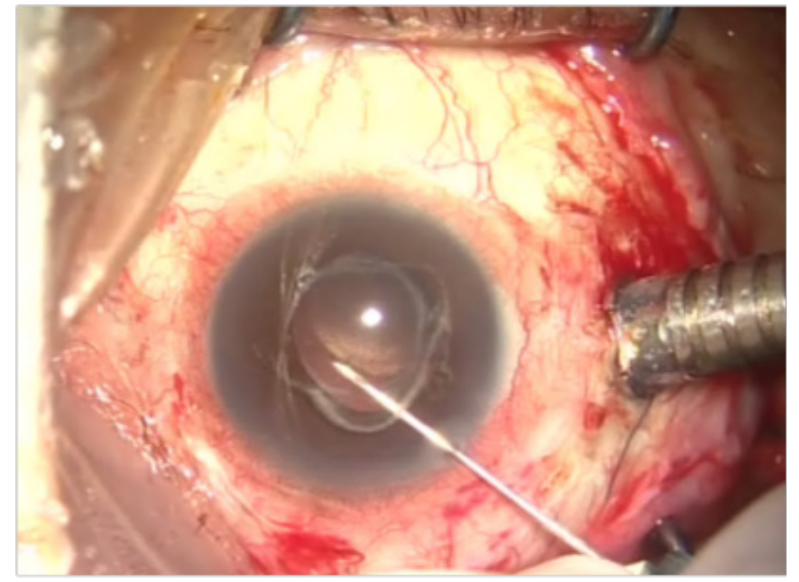

Figure 5 Displacing emulsified oil from the visual axis by healon.

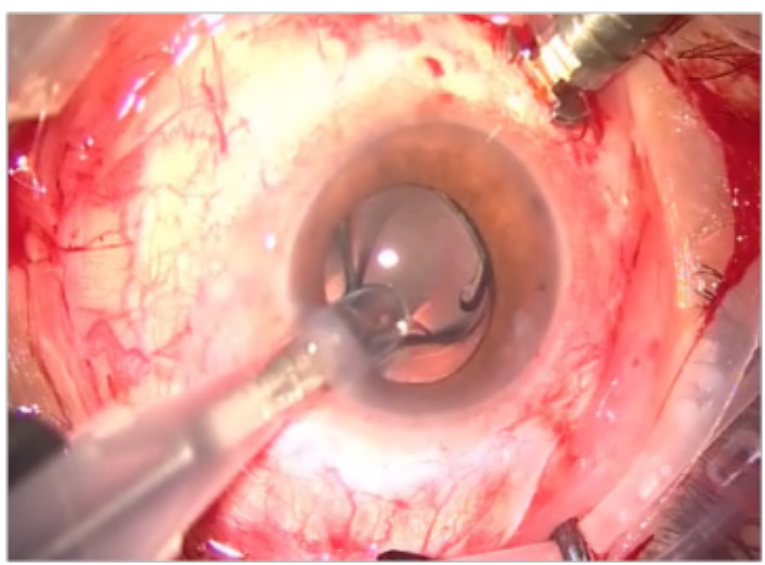

Figure 6 Phacoemulsification \& foldable IOL in bag.

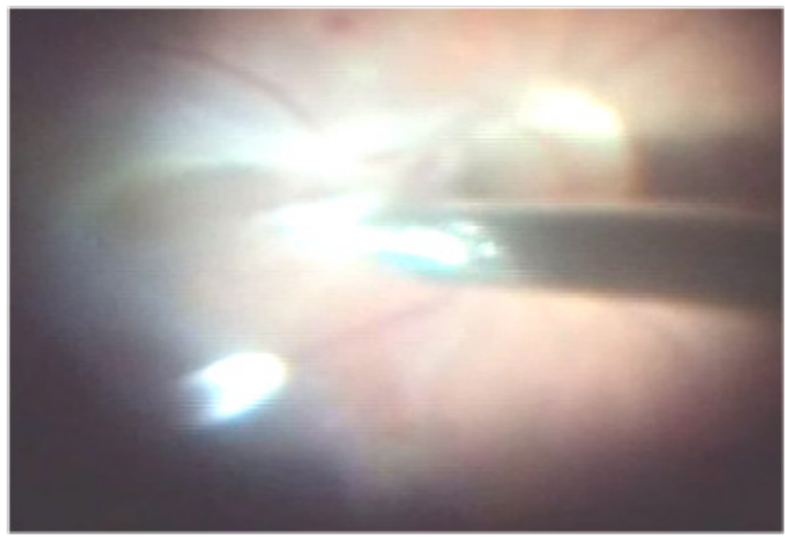

Figure 7 Peeling ERM under silicone oil.

\section{Relaxing retinotomy \& retinectomy}

i. Indicated if extensive retinal shortening persists after membrane removal.

ii. A, Arrows indicate both circumferential and radial retinal shortening. Diathermy applied to retina and vessels in proposed retinotomy site. Retinotomy extended obliquely anteriorly into uninvolved retina to maximize relaxation. iii. B, Retina flat following fluid-silicone exchange. Anterior flap of functionless retina excised, which reduces likelihood of recurrent traction in area of retinotomy. Note bare retinal pigment epithelium (RPE) anterior to retinotomy (Figures 8 \& 9).

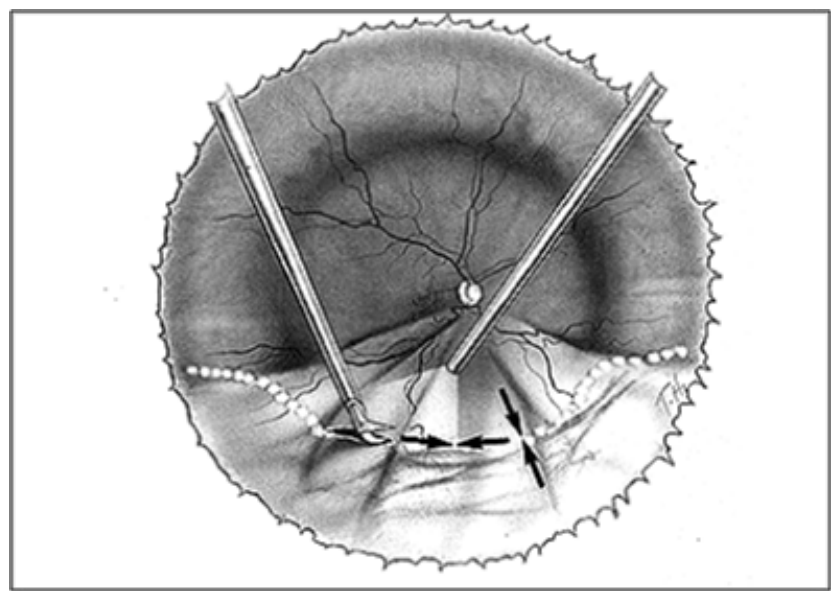

Figure 8 Relaxing retinotomy \& retinectomy.

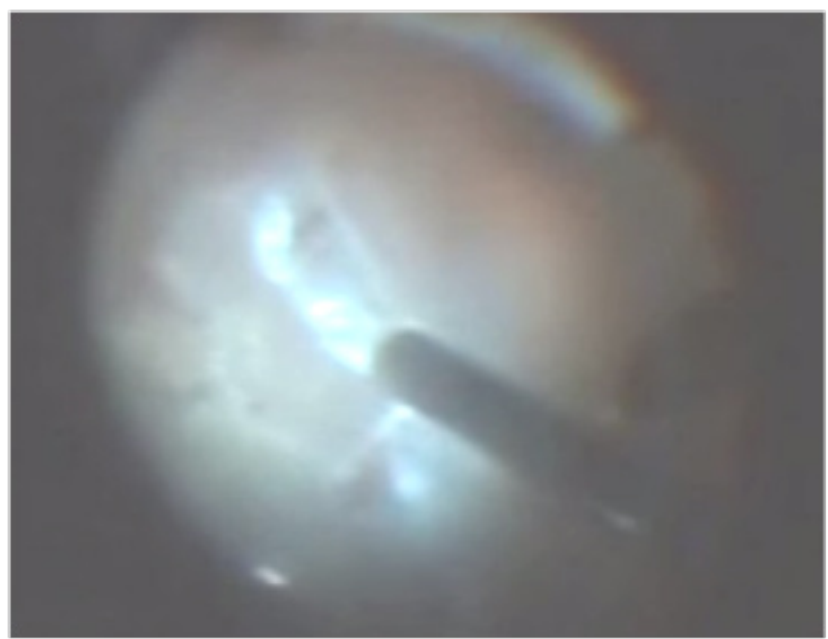

Figure 8 (A) Relaxing retinotomy. Diathermy first done to mark the edge of retinotomy \& decrease bleeding.

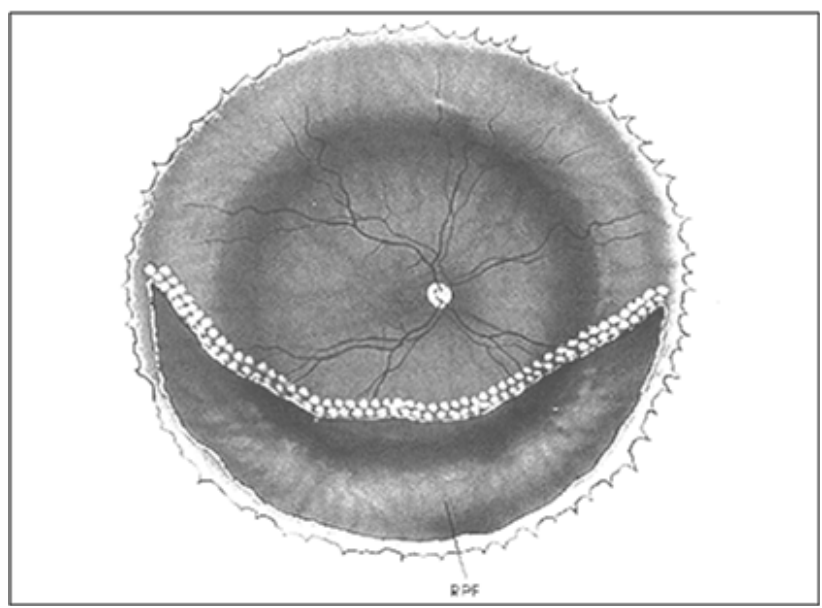

Figure 9 Relaxing retinotomy \& retinectomy. 


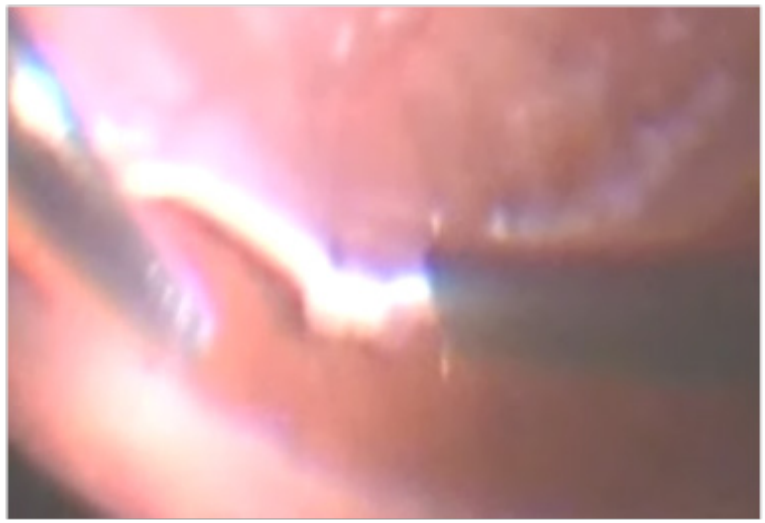

Figure 9 (A) Retinectomy of the anterior retinal flap up to ora under silicone oil.

\section{Results}

i. Anatomical and visual results depended on the cause, extent and duration of retinal detachment and the presence or absence of PVR and its extent.

ii. The 6 cases with an unsupported lower retinal break with no PVR, whether those treated by face down positioning and laser or those treated with hydraulic reattachment, all had $100 \%$ anatomical success from a single intervention. The visual acuity of these cases ranged from $6 / 12$ to $6 / 24$ corrected vision. All the cases had silicone oil removal 3-5 months later with stabilized anatomical and visual functions.

iii. The remaining 44 cases included 3 cases of intrinsic retinal contraction, 34 cases of posterior PVR and 7 cases of combined anterior and posterior PVR.

iv. Cases of intrinsic retinal contractionwere diagnosed intraoperatively during fluid silicone exchange as a part of hydraulic reattachment procedure, and a lower retinectomy, around 180 degrees was done for all 3 cases with laser to the edge of the posterior retina. Anatomical success was initially $100 \%$ but recurrence from the ends of the retinotomy site occurred in one case and needed an additional extension of the retinotomy. Silicone oil was removed uneventfully from all 3 cases 3-5 months later. Corrected visual acuity in this group ranged between $6 / 36 \& 6 / 60$.

i. In the 34 cases of posterior PVR anatomical success from a single intervention occurred in 20 eyes $(58.8 \%)$ and from more than one intervention in 9 eyes $(26.5 \%)$ and failure to reposite the retina in a permanent manner occurred in the remaining 5 eyes (14.7\%) .

ii. Cases of focal PVR with a limited retinal elevation of 4 or less clock hours had the best prognosis and retina flattened from a single intervention. As regards the visual function in this group, it ranged from $6 / 12$ to $6 / 36$ corrected vision. The vision was better in the cases where the focal star folds were away from the fovea. In this group, silicone oil was removed 3-5 months later and the retina remained flat in 17 of the 20 cases $(85 \%)$. The retina was redetached in the remaining 3 cases $(15 \%)$ and silicone oil was reinjected and the retina successfully flattened.

iii. In the 9 eyes that needed multiple surgical interventions to reposite the retina, the posterior PVR was of the diffuse type, the membranes were moderately adherent to the underlying retina and the extent of detachment was 180 degrees or more. Silicone oil was not removed from these eyes and the final visual acuity ranged from $6 / 60$ to $1 / 60$.

iv. All 5 cases of failure were those with tough diffuse fibrous tissue , usually bridging over major retinal vessels and intraoperative bleeding and/or iatrogenic breaks usually occurred while removing the epiretinal membranes.

v. In the 7 cases of combined anterior and posterior PVR, anatomical success was achieved in 4 cases (57.1\%) after multiple surgical interventions, and failure to reposite the retina permanantly occurred in 3 cases $(42.9 \%)$. In the first 4 cases silicone oil was not removed, and a final visual acuity of 5/60 to $1 / 60$ was achieved.

\section{Discussion}

In our study, we managed cases of recurrent retinal detachment under silicone oil due to an unsupported lower break with no PVR by face down positioning followed by laser barrage, and in cases where this technique failed we used hydraulic reattachment. An alternative technique mentioned by Steel et al 1997 \& Raman et al 2010 is to do a laser barrage around the detached area. The rationale is to delimit the subretinal fluid so that the retina remains attached after the removal of silicone oil. In our opinion, however, complete removal of

i. Subretinal fluid and proper apposition of the neurosensory retina to the underlying RPE before laser application in a more radical and effective line of treatment.

ii. Other authors ${ }^{8,7}$ however suggested the use of scleral buckling for these cases of leaking inferior retinal break, with no PVR. In this technique, they did a limited conjunctival opening, the retinal break was localized, and cryotherapy was applied. This was followed by draining the subretinal fluid. Our techniques are, however as effective as this technique, with a less invasive intervention particularly avoiding external drainage of subretinal fluid. The hydraulic reattachment technique also provides an effective tool for the diagnosis and management of intrinsic retinal contraction in a more long term efficient way.

iii. In recurrent cases with PVR, we operated in all cases under silicone oil. Other authors, however operated in all cases after silicone oil removal. ${ }^{9}$ Their surgical technique included evacuation of silicone oil, TA assisted search for residual posterior cortical vitreous, epiretinal membrane peeling, stabilization of posterior retina with PFCL, excision and dissection of basal vitreous gel and peripheral membranes, retinotomy/retinectomy $(180-360$ degrees), removal of subretinal proliferation, complete retinal reattachment with PFCL, endolaser and finally direct PFCL/ Silicone oil exchange. We believe that our technique provides the same facilities for posterior membrane dissection. Anterior PVR in these cases is usually very advanced and the membranes very tough and in most cases impossible to dissect, so a retinectomy in our hands provides more anatomical success and this can be done readily under silicone oil. The only disadvantage of our technique is when bleeding occurs. It is, in this case better to avoid by adequate diathermy to the edges of the retinotomy and more aggressively at the sites where major blood vessels are present. If bleeding occurs, injection of additional silicone oil to increase 
the intraocular pressure temporarily is the best method to stop

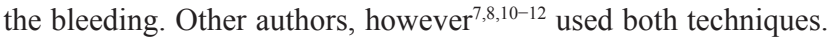
They reserved the technique where silicone oil was to be removed as a first step for cases of recurrence of retinal detachment in phakic eyes \& combined anterior and posterior PVR \& cases with extensive subretinal proliferation.

iv. Anatomical success in our series ranged from $100 \%$ in cases with an unsupported break with no PVR to $85.2 \%$ in cases of retinal detachment with posterior PVR to $57.1 \%$ in cases of combined anterior and posterior PVR.

v. Our overall anatomical success was $84 \%$ which is close to the $89 \%$ reported by Mortada ${ }^{11}$ and better than that reported by Sharma et al. ${ }^{12}(67 \%)$, Nawrocki \&Starska $(76.8 \%)$ and Raman et al. ${ }^{10}$ $(66 \%)$. The analysis of the different cases as regards the extent of detachment, the presence or absence of PVR and the extent and location of PVR may be responsible for the variable results.

vi. As regards the visual function, vision improved in our series to levels ranging from $6 / 12$ to $1 / 60$ according to the degree of pathology present. Vision was best in cases with no PVR followed by cases of intrinsic retinal contraction and focal posterior PVR away from the fovea, followed by diffuse posterior PVR and worst with combined anterior and posterior PVR. These results are similar to the results mentioned by other authors. ${ }^{12}$

vii. The main complications we encountered intraoperatively were bleeding from retinotomy site in 17 cases (34\%) and iatrogenic breaks while peeling tough ERMs in 3 cases $(6 \%)$. Postoperatively we had hypotony in 5 cases $(10 \%)$ and corneal decompensation in 3 cases $(6 \%)$. These complications are similar to those mentioned by Mortada ${ }^{11}$ who reported persistent hypotony in $9 \%$, increased IOP in $11 \%$ and corneal decompensation in $5 \%$ of cases in his series.

\section{Acknowledgments}

None.

\section{Conflicts of interest}

Author declares that there is no conflict of interest.

\section{References}

1. Jonas JB, Knorr HL, Rank RM, et al. Retinal redetachment after removal of intraocular silicone oil tamponade. $\mathrm{Br} J$ Ophthalmol. 2012;85(10):1203-1207.

2. Asaria RH, Gregor ZJ. Simple retinal detachments: identifying the at-risk case. Eye(Lond). 2002;16(4):404-410.

3. The Silicone Study Group. Vitrectomy with silicone oil or perfluoropropane gas in eyes with severe proliferative vitreoretinopathy: results of a randomized clinical trial. Silicone Study Report 2. Arch Ophthalmol. 1992;110(6):780-792.

4. The Silicone Study Group. Vitrectomy with silicone oil or sulfur hexafluoride gas in eyes with severe proliferative vitreoretinopathy: results of a randomized clinical trial. Silicone Study Report 1. Arch Ophthalmol. 1992;110(6):770-779.

5. Lewis H, Burke JM, Abrams GW, et al. Perisilicone proliferation after vitrectomy for proliferative vitreoretinopathy. Ophthalmology. 1988;95(5):583-591.

6. Kapran Z, Uyar OM, Kaya V, et al. Recurrences of retinal detachment after vitreoretinal surgery, and surgical approach. Eur J Ophthalmol. 2001;11(2):166-170.

7. Kamal_A_M_Solaiman.

8. Sharma YR, Karunanithi S, Azad RV, et al. Functional and anatomic outcome of scleral buckling versus primary vitrectomy in pseudophakic retinal detachment. Acta Ophthalmol Scand. 2005;83(3):293-297.

9. http://www.evrs.eu/recurrent-retinal-detachment-in-silicone-oil-filledeyes/Mortada,H

10. Raman R, Sharma T. Management of recurrent retinal detachment in silicone oil-filled eyes. Tech Ophthalmology. 2010;8:122-126.

11. Steel DH, Weir P, James CR. Silicone assisted, argon laser confinement of recurrent proliferative vitreoretinopathy related retinal detachment: a technique to allow silicone oil removal in problem eyes. Br J Ophthalmol. 1997;81(9):765-770.

12. Sharma T, Gopal L, Shanmugam MP, et al. Management of recurrent retinal detachment in silicone oil-filled eyes. Retina. 2002;22(2):153-157. 